\title{
Generalized Spatial Differentiation from the Spin Hall Effect of Light and Its Application in Image Processing of Edge Detection
}

\author{
Tengfeng Zhu, ${ }^{1,+}$ Yijie Lou, ${ }^{1}, \stackrel{+}{\ddagger}$ Yihan Zhou, ${ }^{1}$ Jiahao Zhang, ${ }^{1}$ Junyi Huang, ${ }^{1}$ Yan Li, ${ }^{3}$ Hailu Luo, ${ }^{4}$ \\ Shuangchun Wen, ${ }^{4}$ Shiyao Zhu, ${ }^{1,2}$ Qihuang Gong, ${ }^{3}$ Min Qiu, ${ }^{2,{ }^{*}}$ and Zhichao Ruan ${ }^{1,2, \dagger}$ \\ ${ }^{1}$ Zhejiang Province Key Laboratory of Quantum Technology and Device, Department of Physics and State Key \\ Laboratory of Modern Optical Instrumentation, Zhejiang University, Hangzhou 310027, China \\ ${ }^{2}$ State Key Laboratory of Modern Optical Instrumentation and College of Optical Engineering, \\ Zhejiang University, Hangzhou 310027, China \\ ${ }^{3}$ State Key Laboratory for Mesoscopic Physics and School of Physics, Peking University, \\ Beijing 100871, China \\ ${ }^{4}$ Key Laboratory for Micro/Nano Optoelectronic Devices of Ministry of Education, \\ School of Physics and Electronics, Hunan University, Changsha 410082, China
}

(Received 23 July 2018; revised manuscript received 25 November 2018; published 18 March 2019)

\begin{abstract}
Optics naturally provides us with some powerful mathematical operations. Here, we experimentally demonstrate that during reflection or refraction at a single optical planar interface, the optical computing of spatial differentiation can be realized by analyzing specific orthogonal polarization states of light. We show that the spatial differentiation is intrinsically due to the spin Hall effect of light and generally accompanies light reflection and refraction at any planar interface, regardless of material composition or incident angles. The proposed spin-optical method takes advantages of a simple and common structure to enable vectorialfield computation and perform edge detection for ultrafast image processing.
\end{abstract}

DOI: 10.1103/PhysRevApplied.11.034043

\section{INTRODUCTION}

Planar interfaces between two isotropic materials are the simplest optical structures but fundamentally reveal the nature of wave optics, such as Snell's law, Fresnel coefficients, and the Brewster angle. Several decades ago, it was observed that optical beams totally reflected by planar interfaces exhibit different transverse shifts dependent on polarization state $[1,2]$. However, until recently, such an effect was first theoretically studied in the context of a geometric phase (Berry phase) [3-6] and later as total angular momentum conservation $[7,8]$. These theories greatly deepen the understanding of the spin Hall effect (SHE) of light: the spin-dependent transverse shift generally accompanies light reflection and refraction at planar interfaces and is geometrically protected by the RytovVladimirskii-Berry phase [9], which is distinct from the

\footnotetext{
*minqiu@zju.edu.cn

${ }^{\dagger}$ zc.ruan@gmail.com

¥These authors contributed equally to this work.
}

Published by the American Physical Society under the terms of the Creative Commons Attribution 4.0 International license. Further distribution of this work must maintain attribution to the author(s) and the published article's title, journal citation, and DOI.
Goos-Hänchen shift $[10,11]$ and the Pancharatnam-Berry phase [12]. Also, they boost up experimental investigations of SHE of light in a variety of different optical systems [13-23] and the other general spin-dependent transportation phenomena [24-28]. In particular, Hosten and Kwiat identified the connection between the optical SHE of photons and classical light and proposed the "weak measurement" method for attaining ultrahigh sensitivity to angstrom-scale displacements [13]. We note that, currently, extensive investigations of the SHE of light are focused on analyzing nonorthogonal polarization states between incident and reflected (refracted) light in order to realize weak measurement. A few studies are involved in orthogonal polarization analysis, which creates a profoundly different output beam profile from the original one $[15,16,18,29]$.

In this paper, we experimentally demonstrate that under paraxial approximation, by analyzing specific orthogonal polarization states, the beam profiles reflected and refracted at a single optical planar interface correspond to spatial differentiation of incident field. Although the resulting spatial differentiation seems counterintuitive to the common knowledge that no light can pass through two orthogonal polarizers, we show that the spatial differentiation is intrinsically due to the SHE of light and occurs at any planar interface, regardless of material composition 
or incident angles. Here, our theoretical and experimental works fill the gap from observing a few specific cases [15, $16,18,29]$ to confirming generalized optical computing.

Moreover, the spin-optical spatial differentiation scheme offers a robust image processing to extract the boundary of objects, where two different images can be stored in two different polarization states. We note that the optical analog computing of spatial differentiation has been of great interest because it enables massively parallel processing to an entire image in a single shot. This operation is ultrafast in comparison with the standard digital electronic processing [30,31] and attractive for real-time image processing in medical and satellite applications $[32,33]$.

Traditionally, edge contrast enhancement is applied to phase contrast microscopy by shifting the phase of the zero-order Fourier component of the object wave [34]. Later, the Nomarski prism method [35] and the spiral phase filter method based on Fourier optics [36-41] were developed to strongly amplify the image intensity at edges, where the output field is related to the differentiation or the gradient of the incident field. Recently, significant efforts have been made to shrink the thickness of optical computing elements down to the single-wavelength or even subwavelength scale [42-62]. In particular, Silva et al. theoretically proposed a complex array of metaatoms to realize desired mathematical operations [42]. Further within the subwavelength scale, it was experimentally demonstrated that a 50-nm-thick silver layer can realize spatial differentiation by exciting surface plasmon polaritons [53]. Here, without complex Nomarski prisms [35] and filter-based Fourier optics [36-41], the spinoptical scheme offers a simple but powerful mechanism to process the vectorial field with even thin and common structure, a single planar interface. Furthermore, unlike the miniaturization methods based on resonant effects, such as the surface plasmonic method [53], the SHE of light is a nonresonant effect. Correspondingly, the frequency bandwidth of the spatial differentiation computing is unlimited, which enables an optically fast operation speed.

\section{SPATIAL DIFFERENTIATION FROM SPIN HALL EFFECT OF LIGHT}

To understand the generalized spatial differentiation, we first consider spin-1 photons through a preparationpostselection process. The initial state is $\left|\Psi_{\text {in }}\right\rangle=\left|\varphi_{\text {in }}\right\rangle|s\rangle$, where $\left|\varphi_{\text {in }}\right\rangle$ denotes the initial wave function $\left|\varphi_{\text {in }}\right\rangle=$ $\int d k_{y} \tilde{\varphi}_{\text {in }}\left(k_{y}\right)\left|k_{y}\right\rangle=\int d y \varphi_{\text {in }}(y)|y\rangle$ and $|s\rangle$ corresponds to the particle's spin state. Because of the coupling between the spin and the transverse momentum, the spin Hall effect represents the wave packet split for the parallel spin state $|+\rangle$ (left-circularly polarized, $s=+1$ ) and the antiparallel spin state $|-\rangle$ (right-circularly polarized, $s=-1$ ) with a shift in the opposite direction: $\left|\Psi_{\text {out }}\right\rangle=\hat{U}\left|\Psi_{\text {in }}\right\rangle=$ $\int d k_{y} \tilde{\varphi}_{\text {in }}\left(k_{y}\right) \exp \left(i k_{y} \hat{\sigma}_{3} \delta\right)\left|k_{y}\right\rangle| \pm\rangle=\int d y \varphi_{\text {in }}(y \pm \delta)|y\rangle| \pm\rangle$. Here, $\hat{U}$ is the evolution operator, $\hat{\sigma}_{3}$ is the Pauli matrix along $z$ and $\hat{\sigma}_{3}| \pm\rangle= \pm| \pm\rangle$. $\exp \left(i k_{y} \hat{\sigma}_{3} \delta\right)$ represents a coupling between the spin and the transverse momentum of the spin particles, and hence the wave packets of the parallel and antiparallel spin states shift $-\delta$ and $+\delta$, respectively.

Although $\pm \delta$ for the parallel and antiparallel spin states have been extensively investigated in the context of the weak measurement, here we propose an orthogonal polarization analysis to enable destructive interference between two spin states [cf. the minus sign in Eqs. (1) and (3)], which is one of the main points of the present study. In the special case, when we prepare the initial spin state as $|u\rangle=(1 / \sqrt{2})(|+\rangle+|-\rangle)$ and postselect the orthogonal spin state $|v\rangle=(1 / \sqrt{2} i)(|+\rangle-|-\rangle)$, the final measured wave function is left as

$$
\begin{aligned}
\left|\varphi_{\text {out }}\right\rangle & =\left\langle v|\hat{U}| \varphi_{\text {in }}\right\rangle|u\rangle \\
& =\frac{i}{2} \int d k_{y} \tilde{\varphi}_{\text {in }}\left(k_{y}\right)\left(e^{i k_{y} \delta}-e^{-i k_{y} \delta}\right)\left|k_{y}\right\rangle \\
& =\frac{i}{2} \int d y\left[\varphi_{\text {in }}(y+\delta)-\varphi_{\text {in }}(y-\delta)\right]|y\rangle .
\end{aligned}
$$

Therefore, if the initial wave function profile is much larger than the shift $\delta$, the final output wave function $\left|\varphi_{\text {out }}\right\rangle$ is approximately proportional to the first-order spatial differentiation of the input wave function:

$$
\left|\varphi_{\text {out }}\right\rangle \simeq i \delta \int d y \xi(y)|y\rangle
$$

with $\xi(y)=d \varphi_{\text {in }}(y) / d y$. As shown in Eq. (1), the spatial differentiation results from the opposite shifts between the parallel and antiparallel spin states, and the postselected state is orthogonal to the initial one in order to enable destructive interference. Straightforwardly, such a spatial differentiation can also be achieved by preparing the initial spin state $|v\rangle$ and postselecting the orthogonal spin state $|u\rangle$.

The principle of the spatial differentiation from the photonic SHE can be adopted in the classical level with a large number of photons in a quantum-mechanical coherent state, where each photon behaves independently and the light is treated coherently in the paraxial regime. To show the generality of the spatial differentiation, we consider a single optical interface between two isotropic media shown in Fig. 1. Since the SHE of light appears in both reflection and refraction $[13,16]$, our discussion below focuses on the reflection case and the conclusion can be straightforwardly extended to the refraction case.

Suppose that a paraxial beam obliquely illuminates on the interface and the incident and reflected beams have the vectorial electric fields $\mathbf{E}_{\text {in }}$ and $\mathbf{E}_{\text {out }}$ dominating in the transversal direction. We first consider 


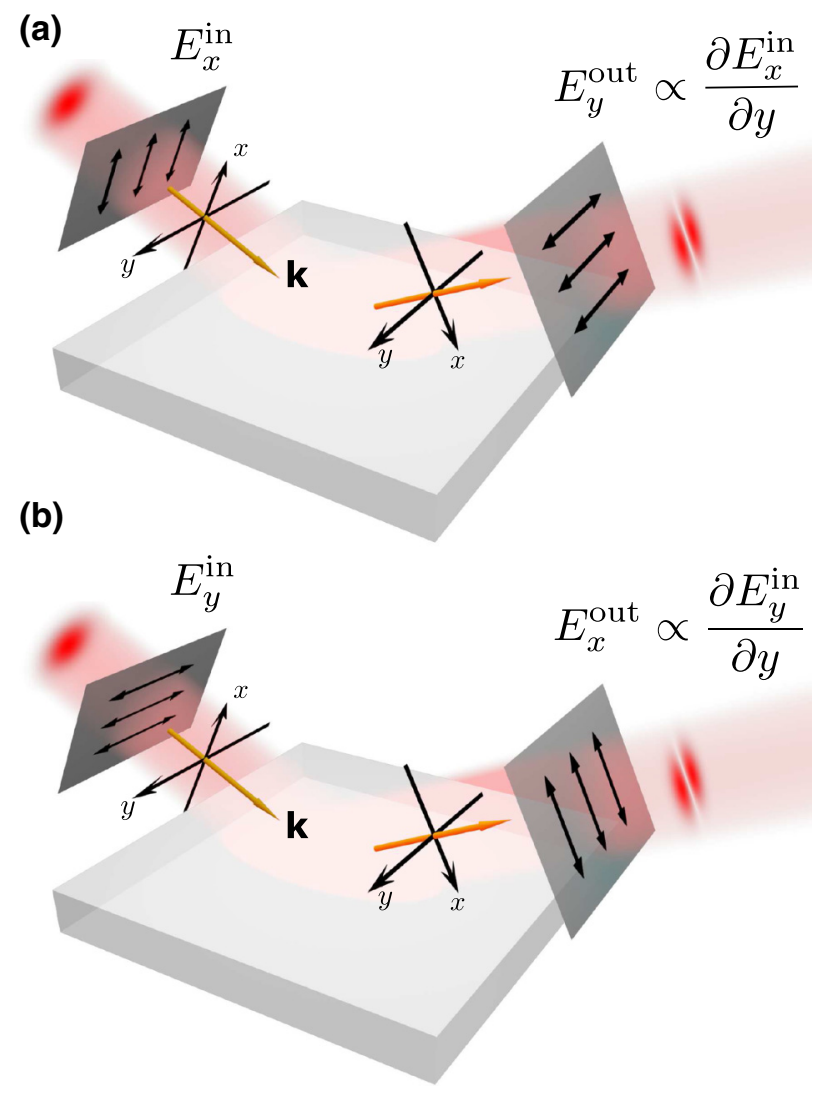

FIG. 1. Schematic of spatial differentiation from the SHE of light on an optical planar interface between two isotropic materials, e.g., an air-glass interface. The two polarizers (dark gray) are oriented at the angles indicated with double-head arrows: (a) preparing along $x$ and analyzing along $y$ and (b) vice versa. As a result, when an obliquely incident paraxial beam has an electric field distribution of $f(x, y)$, the output field distribution corresponds to $\partial f / \partial y$. Here, $x$ and $y$ are the beam profile coordinates for the incident and reflected light, which are perpendicular to the beam propagation direction as the wavevectors $\mathbf{k}$ and share the same origin on the interface, and $x$ is in the incident plane.

the incident and reflected electric fields along $x$ and $y$, respectively, as schematically shown in Fig. 1(a): $\mathbf{E}_{\text {in }}=\mathbf{u}_{x} E_{x}^{\text {in }}(x, y)$ and $\mathbf{E}_{\text {out }}=\mathbf{u}_{y} E_{y}^{\text {out }}(x, y)$, where $\mathbf{u}_{x}$ and $\mathbf{u}_{y}$ correspond to the $x$ - and $y$-unit vectors, respectively. By a vectorial spatial Fourier transform, the incident (reflected) beam can be written as the superposition of plane waves; $E_{x}^{\text {in }}$ and $E_{y}^{\text {out }}$ are written as $E_{x(y)}^{\text {inout })}=$ $\iint \tilde{E}_{x(y)}^{\text {in(out) }}\left(k_{x}, k_{y}\right) \exp \left(i k_{x} x\right) \exp \left(i k_{y} y\right) d k_{x} d k_{y}$. Because of the continuous condition of the tangential wavevector along the interface, the incident plane wave with $\left(k_{x}, k_{y}\right)$ only generates the reflection plane wave with the same $\left(k_{x}, k_{y}\right)$. Therefore, the spatial transform between the incident and reflected electric fields is determined by a spatial spectral transfer function $H\left(k_{x}, k_{y}\right) \equiv$ $\tilde{E}_{y}^{\text {out }}\left(k_{x}, k_{y}\right) / \tilde{E}_{x}^{\text {in }}\left(k_{x}, k_{y}\right)$. Under the paraxial approximation, the spatial spectral transfer function is simplified as (see the detailed derivation within Sec. 2 of the Supplemental Material [63])

$$
H=\frac{i\left(r_{s}+r_{p}\right)}{4}\left(e^{i k_{y} \delta}-e^{-i k_{y} \delta}\right),
$$

where $r_{p}$ and $r_{s}$ are the Fresnel's reflection coefficients of the $p$ and $s$ polarizations for the beam incident angle $\theta_{0}$. In Eq. (3), the two different exponential terms describe the different geometric phases experienced by the leftand the right-handed circular polarizations, resulting in the opposite transverse shifts $[7,8,64] . \delta=2 \cot \theta_{0} / k_{0}$ corresponds to the transverse shift when the incident light is left- or right-handed and the reflected light is analyzed with the same polarization, where $k_{0}$ is the wavevector in the incident medium and the factor of 2 is contributed from the twice coordinate basis rotations during the reflection process. Importantly, the minus sign between two exponential terms in Eq. (3) indicates that, by analyzing polarization orthogonal to the initial one, the reflected filed is the destructive interference between the electric field components.

We note that although a spatial shift can also be observed in other spatial dispersion effects $[10,12]$, it is the minus sign realized by analyzing orthogonal polarization states that acts as the dominant requirement for spatial differentiation. Further with the requirement of $\delta\left|k_{y}\right| \ll 1$, Eq. (3) is approximated as

$$
H \simeq-\frac{\delta\left(r_{s}+r_{p}\right)}{2} k_{y},
$$

which is the transfer function of a first-order $y$-directional spatial differentiator. Correspondingly, in the spatial domain, the reflected $E_{y}^{\text {out }}(x, y)$ field has

$$
E_{y}^{\text {out }}=\frac{i \delta\left(r_{s}+r_{p}\right)}{2} \frac{\partial E_{x}^{\mathrm{in}}}{\partial y}
$$

Equation (5) shows that indeed the spatial differentiation can be realized via the spin Hall effect of light, by a single optical interface. Also, it is straightforward to show that when preparing the $y$-polarized incident fields $\mathbf{E}_{\text {in }}=\mathbf{u}_{y} E_{y}^{\text {in }}$ and analyzing along the $x$ direction $\mathbf{E}_{\text {out }}=\mathbf{u}_{x} E_{x}^{\text {out }}$ [Fig. 1(b)], the reflected electric field corresponds to the spatial differentiation of the incident one as

$$
E_{x}^{\text {out }}=\frac{i \delta\left(r_{p}+r_{s}\right)}{2} \frac{\partial E_{y}^{\text {in }}}{\partial y} .
$$

The invariant transverse direction of the spatial differentiation after switching the orthogonal polarizations can be understood since the circular-polarization-dependent shifts of the SHE occur in the transverse direction. 

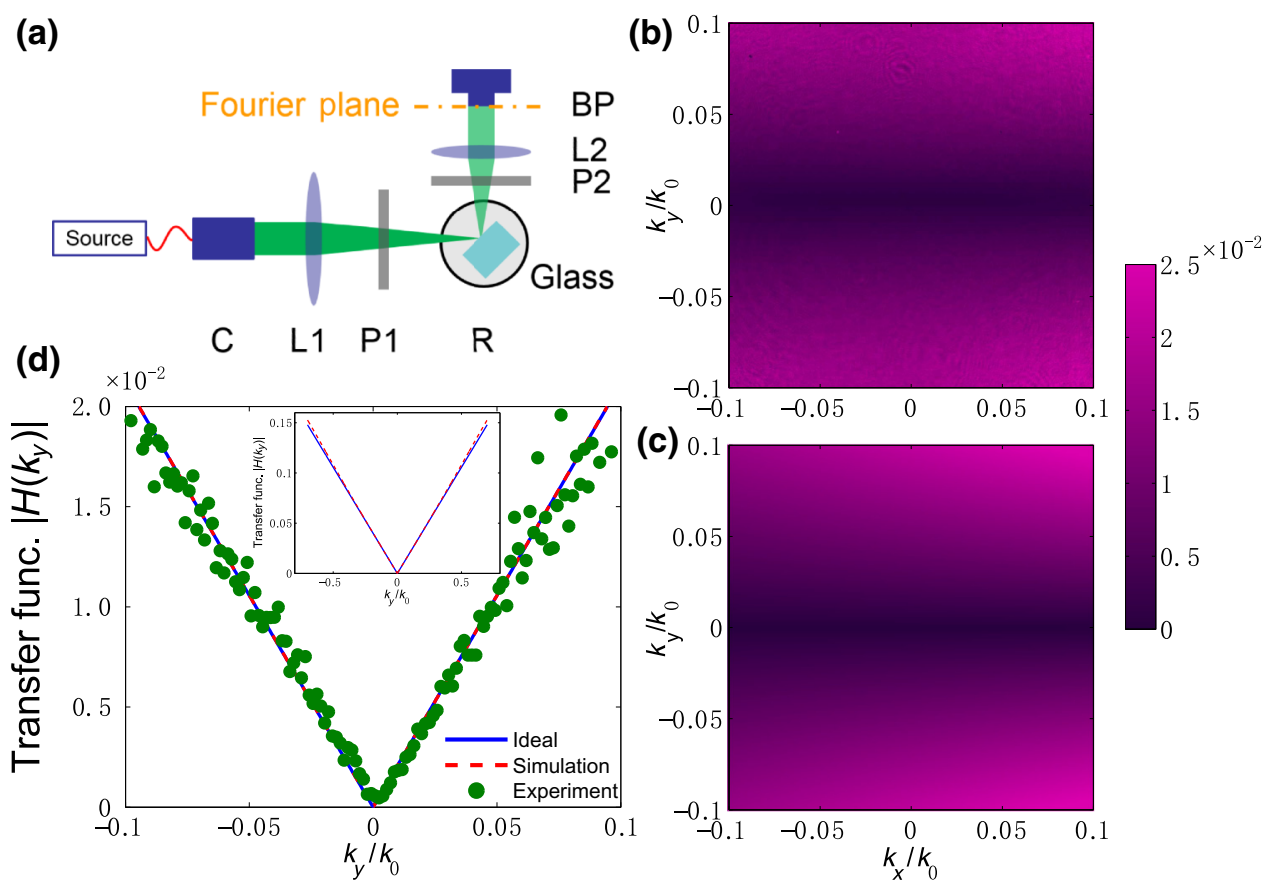

FIG. 2. Measurement of the spatial spectral transfer function on an air-glass interface. (a) Experimental setup: glass slab (material $\mathrm{BK} 7$ ); precision rotator R; polarizers P1 and P2; lenses L1 and L2 with focal lengths 50 and 30 mm, respectively; collimator C; and beam profiler BP (Ophir SP620). The light source is a green laser (wavelength $\lambda_{0}=532 \mathrm{~nm}$ ) and it is connected to the collimator through a fiber with a polarization controller. (b) Measured spatial transfer function spectra with an incident angle $\theta_{0}=45^{\circ}$. (c) Theoretical results of numerical simulations. (d) Experimental (dotted line) and theoretical (dashed lines) spatial spectral transfer function for $k_{x}=0$, and the ideal one (solid lines) based on the first-order differentiation of Eq. (4). The inset corresponds to a wide range from $k_{y}=-0.5 k_{0}$ to $k_{y}=0.5 k_{0}$.

\section{EXPERIMENTAL CHARACTERIZATION AND DEMONSTRATION}

To demonstrate the spatial differentiation, we first measure the spatial spectral transfer function on an air-glass interface, by analyzing the orthogonal polarization. The experiment's setup is schematically shown in Fig. 2(a), where an incident collimated Gaussian laser beam, with the wavelength of $532 \mathrm{~nm}$, focuses on a BK7 glass surface with Lens L1, and the refractive index of the BK7 glass is 1.5195. Lens L2 projects the reflected field to the Fourier space at the back focal plane, which is measured by a beam profiler. The polarizer P1 is used to prepare the initial field polarization state, and the other one $\mathrm{P} 2$ is used to analyze the output polarization state. We note that two polarizers are placed between L1 and L2 in order to avoid the polarization rotation induced by the geometric phase during light focusing and collimating $[65,66]$.

In order to analyze the orthogonal polarization, P1 is placed to make the incident field polarization state along the $x$ direction. The other polarizer $\mathrm{P} 2$ is first along the $y$ direction and the reflected spatial spectra are measured by the beam profiler at the focal plane of L2. The incident spatial spectra are obtained by removing the glass and rotating the reflection path L2 and P2 to the normal incidence.
With the polarizer P2 along the same polarization as $\mathrm{P} 1$, we measure the incident spatial spectra at the back focal plane of L2. Correspondingly, the spatial spectral transfer function is obtained by normalizing the reflected spectrum data with the incident ones. We measure the incident and the reflected spectra with the beam profiler (Ophir SP620) instead of conventional charge-coupled devices (CCDs) because the CCD might use gamma correction and cannot accurately measure the field intensity.

Figure 2(b) shows the measured spatial spectral transfer function for the incident angle $\theta_{0}=45^{\circ}$, where the polarization directions of the polarizer are along the $x$ and $y$ direction, respectively. The measured spatial spectral transfer function is shown in the ranges of $\left|k_{x}\right|<$ $0.1 k_{0}$ and $\left|k_{y}\right|<0.1 k_{0}$, which are limited by the numerical aperture of the optical system. For comparison, Fig. 2(c) corresponds to the theoretical spatial transfer function by numerical simulation (see the details in Sec. 1 of the Supplemental Material [63]). Figures 2(b) and 2(c) show that the experimentally measured spectral transfer function coincides with the theoretical one and has the minimum at $k_{y}=0$. To clearly show the effect of the spatial differentiation, the experimental and numerical transfer functions for $k_{x}=0$ are compared with the ideal firstorder differentiation based on Eq. (4). Indeed, the spatial 


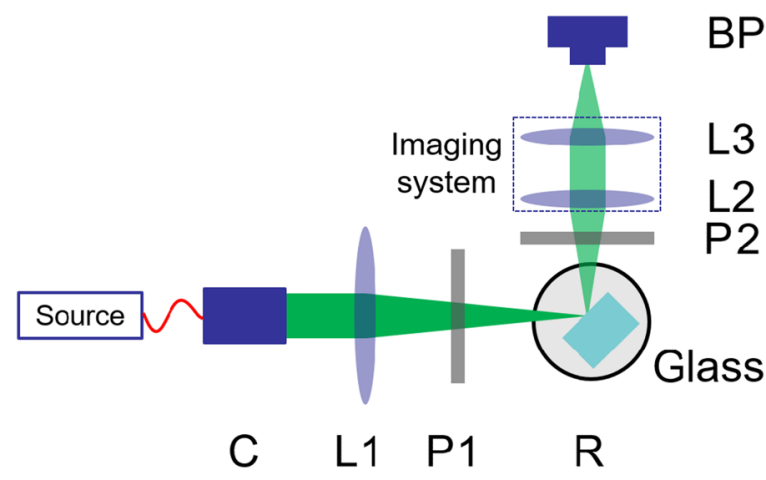

FIG. 3. Experimental setup for spatial differentiation under Gaussian beam illumination. Components include glass slab (material BK7), precision rotator $\mathrm{R}$, polarizers $\mathrm{P}$, lens $\mathrm{L}$, collimator C; and beam profiler BP (Ophir SP620). The light source is a green laser (wavelength $\lambda_{0}=532 \mathrm{~nm}$ ) and it is connected to the collimator through a fiber with a polarization controller.

transfer functions agree well and exhibit a linear dependence of $k_{y}$ around $k_{y}=0$ [Fig. 2(d)]. More importantly, the inset shows that the spatial spectrum bandwidth of the $y$-direction differentiation is as large as about $\left|k_{y}\right|<0.5 k_{0}$.

To illustrate the spin-optical spatial differentiation effect, we measure the reflected field distribution under a Gaussian beam illumination. The setup is shown in Fig. 3, where a collimated green $(532 \mathrm{~nm})$ laser beam illuminates the airglass interface by focusing lens $\mathrm{L} 1$. The reflected beam is projected to the beam profiler with a conjugate imaging system of L2 and L3. The incident angle is controlled with a precision rotator $\mathrm{R}$. Two polarizers $\mathrm{P} 1$ and $\mathrm{P} 2$ are used to enable the spatial differentiation through preparing and analyzing the polarization states in orthogonal directions.

Figures 4(a) and 4(b) show the measured intensity profiles for the incident and reflected beams, respectively. Indeed, the reflected beam exhibits a first-order HermiteGaussian profile with a minimum amplitude at $y=0$, which corresponds to the $y$-direction spatial differentiation of the incident Gaussian field. To quantitatively illustrate the performance of spatial differentiation, we numerically fit the incident beam with a Gaussian profile of a waist radius $w_{0}=44.20 \lambda_{0}$ as illustrated in Fig. 4(c). Figure 4(d) shows the ideal first-order differentiation of Fig. 4(c) in the $y$ direction. In Figs. 4(e) and 4(f), we compare the normalized experimental results with the ideal ones at three different cut-through positions. The dotted and solid lines correspond to the intensities of the experimental and ideal results, respectively. As a direct demonstration of spatial differentiation, the experimental reflected fields show a good agreement with the ideal spatial differentiation in both the peak positions and values [Fig. 4(f)].

\section{APPLICATION IN IMAGE PROCESSING OF EDGE DETECTION}

Since the optical computing of spatial differentiation is important for the ultrafast image processing of edge detection, we demonstrate the spin-optical spatial differentiation in these aspects. We note that the spin-optical method takes advantages of image processing on vectorial fields,
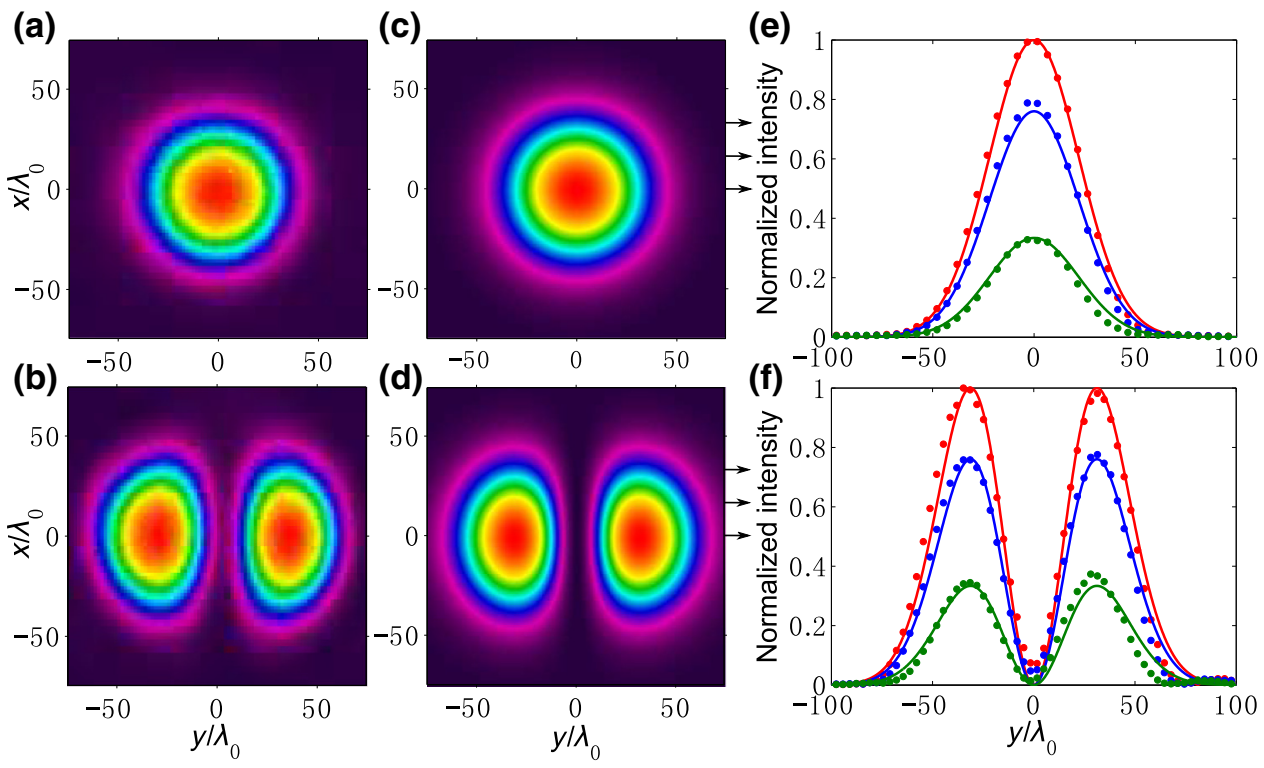

FIG. 4. Spatial differentiation demonstration for a Gaussian illumination with an incident angle $\theta_{0}=45^{\circ}$. (a),(b) Measured intensity profiles of the incident and reflected beams, respectively. (c),(d) Numerical Gaussian fitting (c) to the incident beam with a waist radius $w_{0}=44.2 \lambda_{0}$, and the ideal spatial differentiation results (d) corresponding to $y$-direction differentiation of (c). (e),(f) Normalized intensities of the incident and reflected fields at $x=0.0 \lambda_{0}, 16.54 \lambda_{0}$, and $33.08 \lambda_{0}$, as indicated by the right arrows in (c),(d), where the dotted and solid lines correspond to the intensities of the experimental and ideal results, respectively. 

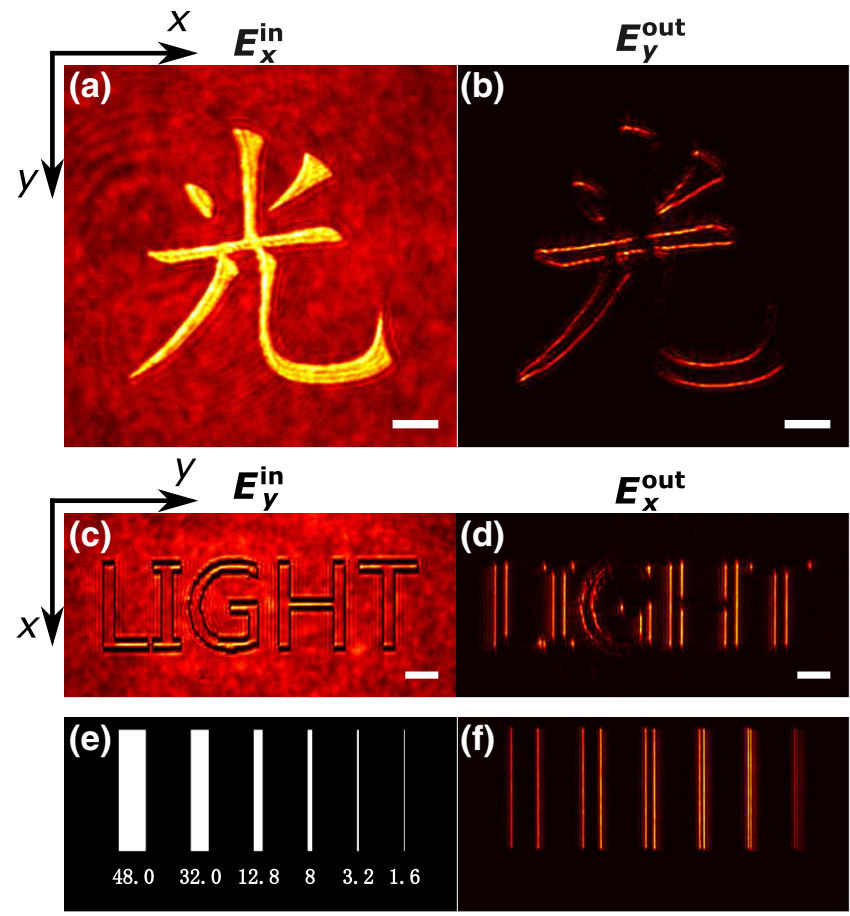

FIG. 5. Edge detection for different images stored in $E_{x}^{\text {in }}$ and $E_{y}^{\text {in }}$, respectively, with either amplitude or phase modulation. (a) Incident image consisting of the Chinese character for "light" with amplitude modulation on $E_{x}^{\text {in }}$. (b) Reflected intensity image corresponding to (a) by measuring $E_{y}^{\text {out }}$. (c) Incident image consisting of the LIGHT letters generated with phase modulation on $E_{y}^{\text {in }}$, where the inside and the outside of the letters have different phases but the same intensity. (d) Reflected intensity image corresponding to (c) by measuring $E_{x}^{\text {out }}$. The white bars correspond to the length of $50 \mu \mathrm{m}$. (e) Slot test patterns on the SLM with the different phases for the black and the white areas. The widths of the slots are listed below in micrometers. (f) Measured reflected intensity image corresponding to (e).

which enables one more degree of freedom than all the current scalar-field schemes [42-57]. As the differentiation operates on the electric field rather than the intensity, the device can be used to detect an edge either in the phase or the amplitude distribution of the incident field. To show such an effect, we use a spatial light modulator (SLM) to generate incident fields with amplitude and phase modulations, respectively. With the experiment setups shown in Fig. S3 of [63], the images are projected to the interface through an imaging system and then are recorded by a beam profiler after differentiation and another imaging system (see the details within Sec. 3 of the Supplemental Material [63]).

Figure 5(a) shows the incident image field consisting of the Chinese character for "light" generated with amplitude modulation on the field component of $E_{x}^{\text {in }}$, where the inside and the outside of the character have different intensities. Figure 5(b) shows the measured reflected intensity of $E_{y}^{\text {out }}$. It clearly exhibits the outlines of the character with spatial differentiation. Since the differentiation is along the $y$ direction, the edges perpendicular to the $y$ direction are most visible. Furthermore, as long as the edge is not completely along the $y$ direction, it can be detected in the reflected beam. Figure 5(c) shows an incident image field of the LIGHT letters generated with phase modulation on the field component of $E_{y}^{\text {in }}$. We note that here we rotate the incident image to demonstrate that we can detect the vertical edges. Again, the reflected light clearly exhibits only the edges of the letters in the vertical direction by measuring $E_{x}^{\text {out }}$ [Fig. 5(d)], which exactly corresponds to spatial differentiation along the $y$ direction. Different from the isotropic edge detection method using spiral phase filters [37-41], the differentiator performs spatial differentiation along only a single direction, which is similar to the Nomarski method [35]. This directional selectivity feature is very useful in image processing to determine and classify edge directions [67].

As shown in Fig. 2(d), since there is a finite spatial bandwidth, the spatial differentiation cannot resolve two edges that are very close to each other. In order to show the edge detection resolution, we generate slot test patterns on (a)

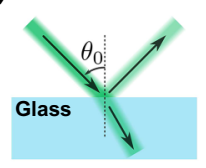

(g)

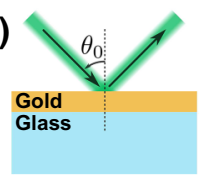

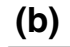

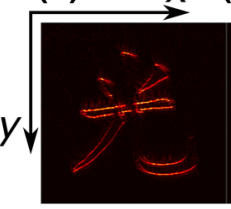

(h)

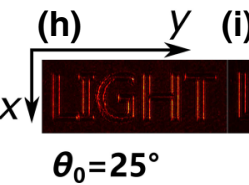

(c)

(d)

(e)

(f)

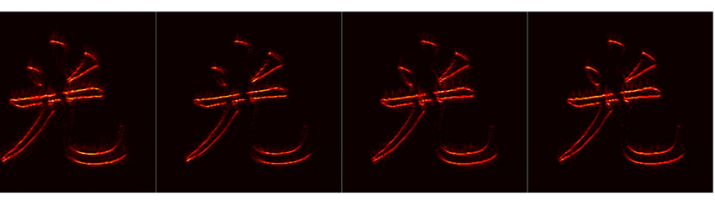

(k)

(I)

(j)

$(\mathbf{k})$
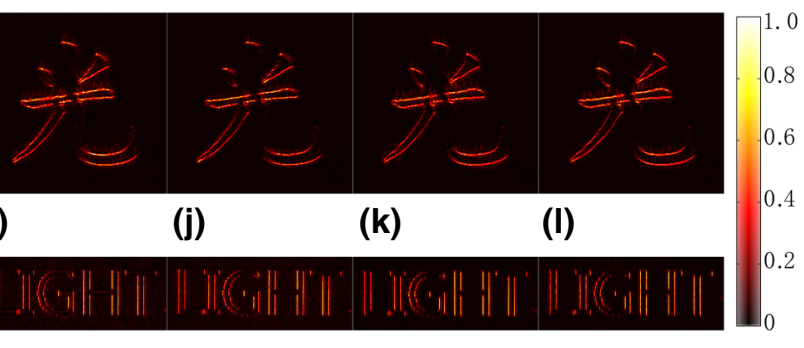

$45^{\circ}$

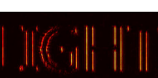

$55^{\circ}$

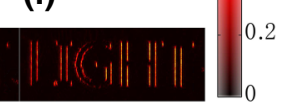

$65^{\circ}$

FIG. 6. Edge detection demonstration on different material interfaces and for a variety of incident angles. (a)-(f) The weak reflection case on the air-glass interface. (g)-(l) The total reflection case with a planar interface on a 210-nm-thick gold layer. (a) and (g) are the schematics of the light reflection for two cases, respectively. The spatial differentiation results are shown for the incident images with (b) (f) the Chinese character for "light" and (h)-(l) the letters LIGHT. Here, the field intensities are normalized with each maximum. The incident angle for each column is listed below. 
the SLM [Fig. 5(e)]. The corresponding reflected intensity is measured as Fig. 5(f), which shows that the minimum separation between the two edges that can be resolved is about $3.2 \mu \mathrm{m}$. The resolution is mainly limited by the small numerical aperture of the image system and can be further improved.

To verify the generality of spin-optical spatial differentiation, we also experimentally demonstrate it for different incident angles and materials. Figures 6(b)-6(f) show the spatial differentiation results on the air-glass interface for the image with the Chinese character for "light," when the incident angle is varied from $25^{\circ}$ to $65^{\circ}$ with a step of $10^{\circ}$. They clearly demonstrate that the spatial differentiation occurs for all the different incident angles. Moreover, since the SHE of light on the interface is a geometric effect that is independent of material, we also demonstrate the spatial differentiation in the total reflection case on the 210-nm-thick gold layer. Figures 6(h)-6(l) show the spatial differentiation results for the image consisting of the LIGHT letters with different incident angles. Indeed, these results confirm that the spatial differentiation is generalized, regardless of the composition materials of the planar interface.

\section{CONCLUSION}

We experimentally demonstrate the generality of spatial differentiation based on the SHE of light. The observation of the spin-optical spatial differentiation is dependent on three key elements: oblique incidence, coherent paraxial beam, and polarizer. The SHE of light vanishes at normal incidence for symmetry reasons; therefore, the spatial differentiation signal is decreased with a small value of $\left|r_{s}+r_{p}\right|$ when reducing the incident angle [cf. Eq. (4)]. Moreover, we show that such a spatial differentiation takes advantages of a simple and common structure to enable vectorial-field computation. Because of the generality of SHE, the proposed method paves a way to ultrafast information processing in a variety of optical systems and can even be forwarded to the processing of electron beams.

\section{ACKNOWLEDGMENTS}

We acknowledge Professor Hui Ye for the help with depositing gold layers on the sample. This work is supported by the National Key Research and Development Program of China (Grant No. 2017YFA0205700), National Natural Science Foundation of China (NSFC Grants No. 91850108 and No. 61675179), and Fundamental Research Funds for the Central Universities (Grant No. 2018QNA3005). M.Q. acknowledges the support by the NSFC (Grant No. 61425023). Y. Li and Q.G. acknowledge the support by the NSFC (Grants No. 11474010 and No. 61590933). H.L. and S.W. acknowledge the support by the NSFC (Grant No. 11474089).
[1] F. I. Fedorov, To the theory of total reflection, Dokl. Akad. Nauk SSR 105, 465 (1955).

[2] C. Imbert, Calculation and experimental proof of the transverse shift induced by total internal reflection of a circularly polarized light beam, Phys. Rev. D 5, 789 (1972).

[3] M. Onoda, S. Murakami, and N. Nagaosa, Hall Effect of Light, Phys. Rev. Lett. 93, 083901 (2004).

[4] K. Y. Bliokh and Y. P. Bliokh, Modified geometrical optics of a smoothly inhomogeneous isotropic medium: The anisotropy, Berry phase, and the optical Magnus effect, Phys. Rev. E 70, 026605 (2004).

[5] K. Y. Bliokh, I. V. Shadrivov, and Y. S. Kivshar, Goos-Hänchen and Imbert-Fedorov shifts of polarized vortex beams, Opt. Lett. 34, 389 (2009).

[6] K. Y. Bliokh and Y. P. Bliokh, Topological spin transport of photons: The optical Magnus effect and Berry phase, Phys. Lett. A 333, 181 (2004).

[7] K. Y. Bliokh and Y. P. Bliokh, Conservation of Angular Momentum, Transverse Shift, and Spin Hall Effect in Reflection and Refraction of an Electromagnetic Wave Packet, Phys. Rev. Lett. 96, 073903 (2006).

[8] K. Y. Bliokh and A. Aiello, Goos-Hänchen and Imbert-Fedorov beam shifts: An overview, J. Opt. 15, 014001 (2013).

[9] S. Rytov, On transition from wave to geometrical optics, Dokl. Akad. Nauk SSSR 18, 263 (1938); V. Vladimirskii, The rotation of a polarization plane for curved light ray, Dokl. Akad. Nauk SSSR 21, 222 (1941); M. Berry, Interpreting the anholonomy of coiled light, Nature 326, 277 (1987).

[10] F. Goos and H. Hänchen, Ein neuer und fundamentaler versuch zur totalreflexion, Ann. Phys. 436, 333 (1947); K. Artmann, Berechnung der seitenversetzung des totalreflektierten strahles, Ann. Phys. 437, 87 (1948); H. K. Lotsch, Beam displacement at total reflection: The Goos-Hänchen effect I, Optik 32, 116 (1970); H. K. Lotsch, Beam displacement at total reflection: The Goos-Hänchen effect II, Optik 32, 189 (1970); H. K. Lotsch, Beam displacement at total reflection-Goos-Hänchen effect III, Optik 32, 299 (1971); H. K. Lotsch, Beam displacement at total reflection-Goos-Hänchen effect IV, Optik 32, 553 (1971).

[11] C. C. Chan and T. Tamir, Angular shift of a gaussian beam reflected near the brewster angle, Opt. Lett. 10, 378 (1985); M. Merano, A. Aiello, M. Van Exter, and J. Woerdman, Observing angular deviations in the specular reflection of a light beam, Nat. Photonics 3, 337 (2009).

[12] S. Pancharatnam, Generalized theory of interference, and its applications, Proc. Indian Acad. Sci.-Sec. A 44, 247 (1956); M. V. Berry, The adiabatic phase and pancharatnam's phase for polarized light, J. Mod. Opt. 34, 1401 (1987).

[13] O. Hosten and P. Kwiat, Observation of the spin Hall effect of light via weak measurements, Science 319, 787 (2008).

[14] K. Y. Bliokh, A. Niv, V. Kleiner, and E. Hasman, Geometrodynamics of spinning light, Nat. Photonics 2, 748 (2008). 
[15] Y. Qin, Y. Li, X. Feng, Y.-F. Xiao, H. Yang, and Q. Gong, Observation of the in-plane spin separation of light, Opt. Express 19, 9636 (2011).

[16] X. Zhou, X. Ling, Z. Zhang, H. Luo, and S. Wen, Observation of spin Hall effect in photon tunneling via weak measurements, Sci. Rep. 4, 7388 (2014).

[17] Y. Qin, Y. Li, X. Feng, Z. Liu, H. He, Y.-F. Xiao, and Q. Gong, Spin Hall effect of reflected light at the air-uniaxial crystal interface, Opt. Express 18, 16832 (2010).

[18] K. Y. Bliokh, C. Samlan, C. Prajapati, G. Puentes, N. K. Viswanathan, and F. Nori, Spin-Hall effect and circular birefringence of a uniaxial crystal plate, Optica 3, 1039 (2016).

[19] H. Luo, X. Ling, X. Zhou, W. Shu, S. Wen, and D. Fan, Enhancing or suppressing the spin Hall effect of light in layered nanostructures, Phys. Rev. A 84, 033801 (2011).

[20] B. Wang, Y. Li, M.-M. Pan, J.-L. Ren, Y.-F. Xiao, H. Yang, and Q. Gong, Spin displacements of a gaussian beam at an air-multilayer-film interface, Phys. Rev. A 88, 043842 (2013).

[21] X. Yin, Z. Ye, J. Rho, Y. Wang, and X. Zhang, Photonic spin Hall effect at metasurfaces, Science 339, 1405 (2013).

[22] P. V. Kapitanova, P. Ginzburg, F. J. Rodrǵuez-Fortuno, D. S. Filonov, P. M. Voroshilov, P. A. Belov, A. N. Poddubny, Y. S. Kivshar, G. A. Wurtz, and A. V. Zayats, Photonic spin Hall effect in hyperbolic metamaterials for polarizationcontrolled routing of subwavelength modes, Nat. Commun. 5, 3226 (2014).

[23] X. Zhou, X. Ling, H. Luo, and S. Wen, Identifying graphene layers via spin Hall effect of light, Appl. Phys. Lett. 101, 251602 (2012).

[24] K. Y. Bliokh, F. Rodrǵuez-Fortuño, F. Nori, and A. V. Zayats, Spin-orbit interactions of light, Nat. Photonics 9 , 796 (2015).

[25] A. Aiello, P. Banzer, M. Neugebauer, and G. Leuchs, From transverse angular momentum to photonic wheels, Nat. Photonics 9, 789 (2015).

[26] F. Cardano and L. Marrucci, Spin-orbit photonics, Nat. Photonics 9, 776 (2015).

[27] S. Xiao, J. Wang, F. Liu, S. Zhang, X. Yin, and J. Li, Spindependent optics with metasurfaces, Nanophotonics 6, 215 (2016).

[28] X. Ling, X. Zhou, K. Huang, Y. Liu, C.-W. Qiu, H. Luo, and $\mathrm{S}$. Wen, Recent advances in the spin Hall effect of light, Rep. Prog. Phys. 80, 066401 (2017).

[29] I. M. Duck, P. M. Stevenson, and E. C. G. Sudarshan, The sense in which a "weak measurement" of a spin-1/2 particle's spin component yields a value 100, Phys. Rev. D 40, 2112 (1989).

[30] D. R. Solli and B. Jalali, Analog optical computing, Nat. Photonics 9, 704 (2015).

[31] H. J. Caulfield and S. Dolev, Why future supercomputing requires optics, Nat. Photonics 4, 261 (2010).

[32] D. L. Pham, C. Xu, and J. L. Prince, Current methods in medical image segmentation, Annu. Rev. Biomed. Eng. 2, 315 (2000).

[33] R. J. Holyer and S. H. Peckinpaugh, Edge detection applied to satellite imagery of the oceans, IEEE Trans. Geosci. Remote Sens. 27, 46 (1989).
[34] F. Zernike, Phase contrast, a new method for the microscopic observation of transparent objects part II, Physica 9, 974 (1942); F. Zernike, Phase contrast, a new method for the microscopic observation of transparent objects, Physica 9, 686 (1942).

[35] R. Allen, G. David, and G. Nomarski, The zeiss-nomarski differential interference equipment for transmitted-light microscopy, Zeitschrift fur wissenschaftliche Mikroskopie und mikroskopische Technik 69, 193 (1969).

[36] J. W. Goodman, Introduction to Fourier Optics (McGrawhill, New York, NY, 1996), 2nd ed.

[37] J. A. Davis, D. E. McNamara, D. M. Cottrell, and J. Campos, Image processing with the radial hilbert transform: Theory and experiments, Opt. Lett. 25, 99 (2000).

[38] K. Crabtree, J. A. Davis, and I. Moreno, Optical processing with vortex-producing lenses, Appl. Opt. 43, 1360 (2004).

[39] S. Fürhapter, A. Jesacher, S. Bernet, and M. Ritsch-Marte, Spiral phase contrast imaging in microscopy, Opt. Express 13, 689 (2005).

[40] A. Jesacher, S. Fürhapter, S. Bernet, and M. Ritsch-Marte, Shadow Effects in Spiral Phase Contrast Microscopy, Phys. Rev. Lett. 94, 233902 (2005).

[41] X. Qiu, F. Li, W. Zhang, Z. Zhu, and L. Chen, Spiral phase contrast imaging in nonlinear optics: seeing phase objects using invisible illumination, Optica 5, 208 (2018).

[42] A. Silva, F. Monticone, G. Castaldi, V. Galdi, A. Alù, and N. Engheta, Performing mathematical operations with metamaterials, Science 343, 160 (2014).

[43] S. Abdollahramezani, K. Arik, A. Khavasi, and Z. Kavehvash, Analog computing using graphene-based metalines, Opt. Lett. 40, 5239 (2015).

[44] A. Chizari, S. Abdollahramezani, M. V. Jamali, and J. A. Salehi, Analog optical computing based on a dielectric meta-reflect array, Opt. Lett. 41, 3451 (2016).

[45] Y. Hwang, T. J. Davis, J. Lin, and X.-C. Yuan, Plasmonic circuit for second-order spatial differentiation at the subwavelength scale, Opt. Express 26, 7368 (2018).

[46] A. Saba, M. R. Tavakol, P. Karimi-Khoozani, and A. Khavasi, Two dimensional edge detection by guided mode resonant metasurface, IEEE Photonics Technol. Lett. 30, 853 (2018).

[47] A. Pors, M. G. Nielsen, and S. I. Bozhevolnyi, Analog computing using reflective plasmonic metasurfaces, Nano Lett. 15, 791 (2014).

[48] Y. Hwang and T. J. Davis, Optical metasurfaces for subwavelength difference operations, Appl. Phys. Lett. 109, 181101 (2016).

[49] L. L. Doskolovich, D. A. Bykov, E. A. Bezus, and V. A. Soifer, Spatial differentiation of optical beams using phaseshifted Bragg grating, Opt. Lett. 39, 1278 (2014).

[50] N. V. Golovastikov, D. A. Bykov, L. L. Doskolovich, and E. A. Bezus, Spatial optical integrator based on phase-shifted Bragg gratings, Opt. Commun. 338, 457 (2015).

[51] A. Youssefi, F. Zangeneh-Nejad, S. Abdollahramezani, and A. Khavasi, Analog computing by Brewster effect, Opt. Lett. 41, 3467 (2016).

[52] Z. Ruan, Spatial mode control of surface plasmon polariton excitation with gain medium: From spatial differentiator to integrator, Opt. Lett. 40, 601 (2015). 
[53] T. Zhu, Y. Zhou, Y. Lou, H. Ye, M. Qiu, Z. Ruan, and S. Fan, Plasmonic computing of spatial differentiation, Nat. Commun. 8, 15391 (2017).

[54] Y. Fang, Y. Lou, and Z. Ruan, On-grating graphene surface plasmons enabling spatial differentiation in the terahertz region, Opt. Lett. 42, 3840 (2017).

[55] C. Guo, M. Xiao, M. Minkov, Y. Shi, and S. Fan, Photonic crystal slab laplace operator for image differentiation, Optica 5, 251 (2018).

[56] D. A. Bykov, L. L. Doskolovich, A. A. Morozov, V. V. Podlipnov, E. A. Bezus, P. Verma, and V. A. Soifer, Firstorder optical spatial differentiator based on a guided-mode resonant grating, Opt. Express 26, 10997 (2018).

[57] Z. Dong, J. Si, X. Yu, and X. Deng, Optical spatial differentiator based on subwavelength high-contrast gratings, Appl. Phys. Lett. 112, 181102 (2018).

[58] J. Zhang and Z. Ruan, Amplitude scaling and lateral shift of leaky radiation from surface plasmon excitation, J. Opt. Soc. Am. B 36, 451 (2019).

[59] W. Wu, W. Jiang, J. Yang, S. Gong, and Y. Ma, Multilayered analog optical differentiating device: Performance analysis on structural parameters, Opt. Lett. 42, 5270 (2017).

[60] L. L. Doskolovich, E. A. Bezus, N. V. Golovastikov, D. A. Bykov, and V. A. Soifer, Planar two-groove optical differentiator in a slab waveguide, Opt. Express 25, 22328 (2017).
[61] H. Kwon, D. Sounas, A. Cordaro, A. Polman, and A. Alù, Nonlocal Metasurfaces for Optical Signal Processing, Phys. Rev. Lett. 121, 173004 (2018).

[62] E. A. Bezus, L. L. Doskolovich, D. A. Bykov, and V. A. Soifer, Spatial integration and differentiation of optical beams in a slab waveguide by a dielectric ridge supporting high-q resonances, Opt. Express 26, 25156 (2018).

[63] See the Supplemental Material at http://link.aps.org/supple mental/10.1103/PhysRevApplied.11.034043 for the numerical calculation of the spatial spectral transfer function, the derivation of Eq. (3), and the experimental setup for amplitude and phase modulations.

[64] K. Y. Bliokh and Y. P. Bliokh, Polarization, transverse shifts, and angular momentum conservation laws in partial reflection and refraction of an electromagnetic wave packet, Phys. Rev. E 75, 066609 (2007).

[65] K. Y. Bliokh, E. A. Ostrovskaya, M. A. Alonso, O. G. Rodrǵuez-Herrera, D. Lara, and C. Dainty, Spin-toorbital angular momentum conversion in focusing, scattering, and imaging systems, Opt. Express 19, 26132 (2011).

[66] Z. Bomzon and M. Gu, Space-variant geometrical phases in focused cylindrical light beams, Opt. Lett. 32, 3017 (2007).

[67] R. Jain, R. Kasturi, and B. G. Schunck, Machine Vision (McGraw-Hill, New York, 1995), 1st ed. 